Cahiers de recherches médiévales

La réception d'Isidore de Séville durant le Moyen Âge tardif (XII $-\mathrm{XV}^{\mathrm{e}} \mathrm{s}$.)

\title{
Manuscrits copiés en série
}

Les quatre témoins contemporains du Livre des fais et bonnes meurs du sage roy Charles $\mathrm{V}^{\mathrm{e}}$ d'icelluy nom

\section{Gilbert Ouy, Christine Reno et James Laidlaw}

\section{(2) OpenEdition}

\section{Journals}

Édition électronique

URL : https://journals.openedition.org/crm/10922

DOI : $10.4000 / \mathrm{crm} .10922$

ISSN : 1955-2424

Éditeur

Honoré Champion

Édition imprimée

Date de publication : 10 décembre 2008

Pagination : 239-252

ISSN : $1272-9752$

Référence électronique

Gilbert Ouy, Christine Reno et James Laidlaw, " Manuscrits copiés en série », Cahiers de recherches médiévales [En ligne], 16 | 2008, mis en ligne le 15 décembre 2011, consulté le 15 décembre 2022. URL : http://journals.openedition.org/crm/10922 ; DOI : https://doi.org/10.4000/crm.10922 


\title{
酷M
}

\section{Manuscrits copiés en série : les quatre témoins contemporains du Livre des fais et bonnes meurs du sage roy Charles $V^{e} d^{\prime i c e l l u y ~ n o m ~}{ }^{1}$}

\begin{abstract}
This article makes a detailed comparison of the four surviving copies of the Livre des fais et bonnes meurs du sage roy Charles V that were prepared under Christine de Pizan's supervision: Paris, BnF fr. 5025 and 10153, Modena, Biblioteca Estense $\alpha . N .8 .7$ and Vatican City, Vat. Reg. lat. 920. The similarities and differences among the copies allow one to draw some conclusions about work practices in the author's atelier.

Résumé :Cet article présente une comparaison détaillée des quatre exemplaires survivants du Livre des fais et bonnes meurs du sage roy Charles V préparés sous la direction de l'auteur: Paris, BnF fr. 5025 et 10153, Modena, Biblioteca Estense $\alpha . N .8 .7$ et Città del Vaticano, Vat. Reg. lat. 920. Les similitudes et divergences qu'on observe dans ces quatre témoins permettent de formuler quelques conclusions concernant les pratiques de travail dans l'atelier de Christine de Pizan.
\end{abstract}

Dans le Charles $V$, Christine de Pizan raconte comment Philippe de Bourgogne, au début de l'année 1404, lui commanda, par l'intermédiaire de son trésorier, un traittié sur la vie de son frère Charles V, décédé en $1380^{2}$. Elle se rendit au Louvre et fut conduite par deux écuyers auprès du duc et de l'un de ses fils, Antoine, comte de Rethel ${ }^{3}$. Après cet entretien, elle dut se mettre aussitôt au travail, car, dès le 28 avril $1404^{4}$, la première partie du nouvel ouvrage était déjà terminée ; mais le commanditaire était mort la veille ${ }^{5}$. La deuxième partie fut achevée le 20 septembre $^{6}$ et la troisième - la plus longue - le 30 novembre de cette même année ${ }^{7}$. Le $1^{\text {er }}$ janvier 1405, Christine en offrait à Jean duc de Berry un exemplaire relié qui

\footnotetext{
${ }^{1}$ C'est le titre donné dans les quatre manuscrits contemporains survivants; le $s$ manque à la fin de Charles dans le ms. Vat. Reg. lat. 920.

${ }^{2}$ Sur les motivations tant didactiques que politiques de cette commande, voir l'introduction de l'édition critique: Christine de Pisan. Le Livre des fais et bonnes meurs du sage roy Charles $V$, éd. S. Solente, 2 vol., Paris, Champion, 1936-1940 (1, p. xxviii-xxix); ci-après : LFBM numéro de tome, numéro de page (chiffre romain ou arabe selon le cas). Par contre, toute citation de l'œuvre en manuscrit utilisera le terme "chapitre » et se référera à une des 3 parties (I, II, ou III) suivi du numéro (arabe) du chapitre signalé par les rubriques.

${ }^{3}$ LFBM 1, 6-9.

${ }^{4}$ Observation déjà faite $L F B M 1$, xxix.

${ }^{5} L F B M 1,104$. Cette date est donnée dans l'explicit de la partie I dans tous les manuscrits. La partie II débute par une lamentation sur la mort du duc, où Christine évoque le confort, aide et soustenail de vie qu'il avait apportés à son petit colliege veduval; LFBM 1, 111.

${ }^{6}$ LFBM 1, 244 ; cette date est donnée dans l'explicit de la partie II dans deux des manuscrits, Modena, Biblioteca Estense $\alpha . N .8 .7$, fol. $59^{\mathrm{b}}$ et Vat. Reg. lat. 920, fol. $63^{\mathrm{c}}$.

${ }^{7}$ Cette date se trouve dans la rubrique liminaire de tous les manuscrits : $\operatorname{LFBM} 1,1$.
}

Cahiers de Recherches Médiévales, 16, 2008 
n'a pas été retrouvés. Ce ne devait, d'ailleurs, pas être le premier en date des manuscrits de présentation, car il est à peu près certain que l'auteur donna la priorité à Jean sans Peur, qui venait de succéder à son père Philippe le Hardi.

Le volume offert à Jean sans Peur est le BnF fr. 10153 (B), mais il subsiste trois autres exemplaires de présentation: Modena, Biblioteca Estense a.N.8.7 (E), BnF fr. 5025 (M), et Vat. Reg. lat. 920 (C)9. Nous ignorons quels en étaient les destinataires, mais il est difficile d'imaginer que les deux fils de Charles V, Charles VI et Louis d'Orléans, ainsi que Louis de Bourbon, son beau-frère, qui tous trois y ont droit à un chapitre élogieux, aient pu être oubliés dans la distribution ${ }^{10}$. D'autres grands personnages dont elle vante les mérites (au chap. II, 17 : LFBM 1, 176-80), en particulier le connétable Charles d'Albret, qui acquit un exemplaire de l'Advision Cristine $^{11}$, et qui est le dédicataire d'un exemplaire du Débat des deux amans, Bruxelles, KBR 11034, figurent, eux aussi, parmi les possesseurs probables de l'ouvrage.

Les frappantes similitudes entre les quatre manuscrits de présentation parvenus jusqu'à nous - tant en ce qui regarde la préparation que la mise en page ou la décoration - obligent à conclure qu'ils furent copiés en série; en outre, nombre de corrections identiques prouvent que, juste avant d'être offerts, les quatre volumes furent ensemble soumis à une ultime révision ${ }^{12}$. Cependant, ces manuscrits présentent également d'assez nombreuses divergences grâce auxquelles nous pouvons suivre l'évolution du texte et déterminer l'ordre dans lequel furent copiés les quatre témoins.

\footnotetext{
${ }^{8}$ «Un livre en françoys, des Faiz et bonnes meurs du saige Roy Charles, $V^{e}$ roy d'icellui nom, où il a escript au commancement de second fueillet: ses escuiers; couvert de cuir vermeil empraint, à deux fermouers et clos de cuivre ; lequel livre damoiselle Cristine de Pisan donna à mondit Seigneur à estrainnes, le premier jour de janvier l'an mil CCCC et IIII (a. st.) »; J. Guiffrey, Inventaires de Jean duc de Berry (1401-16), Paris : Leroux 1894-1896, t. 2, p. 9, $\mathrm{n}^{\circ} 943$.

${ }^{9}$ Nous utilisons les sigles adoptés par S. Solente dans $L F B M 1$, lxxxv-xcvii.

${ }^{10}$ Il s'agit des chap. I, 16 et II, 12 (LFBM t. 1, p. 47, 141-44) qui traitent du duc de Berry, et I, 2; II, 13 ( $L F B M 1,7$ et 144-52) qui parlent de Philippe le Hardi. Les inventaires des manuscrits de Charles VI ne portent pas trace de cet ouvrage: voir L. Douët d'Arcq, Inventaire de la bibliothèque du roi Charles VI fait au Louvre en 1423 par ordre du régent, Paris, Société des Bibliophiles, 1867. Cet inventaire, qui inclut les prix, est identique pour les titres à celui de 1411 (p. xii-xiii).

${ }^{11} \mathrm{H}$. Stein, "La Bibliothèque du connétable d'Albret à Sully-sur-Loire (1409)», Le Bibliographe Moderne, 6 (1902), p. 91-93. Cet inventaire ne fait aucune mention des Fais et bonnes meurs. Le ms. Bruxelles, KBR 11034, qui renferme le Débat de deux amans, débute par une ballade qui est dédiée au connétable, mais ce manuscrit ne figure pas non plus à l'inventaire de 1409. La mère de Charles d'Albret était Marguerite de Bourbon, sœur de la reine Jeanne de Bourbon, femme de Charles V.

${ }^{12}$ Le fait que l'exemplaire offert à Jean de Berry le $1^{\text {er }}$ janvier 1405 était relié avant la présentation porte à croire que les autres manuscrits l'étaient aussi, mais ce n'est qu'une hypothèse.
} 


\section{Profil des manuscrits}

De ces quatre manuscrits contemporains survivants, trois sont copiés par $R$ et un par $X$, de toute probabilité l'auteur ${ }^{13}$. Nous passons rapidement en revue leurs caractéristiques principales dans l'ordre présumé de leur exécution; ordre dont nous nous expliquerons au fur et à mesure de notre analyse.

1. BnF fr. 10153 $^{14}$ (B). Parchemin, $298 \times 215$ mm; justification $196 \times 133$ mm; 32 ou 33 interlignes. (I) ${ }^{15}+$ I + (II) $+107+$ I + (I) ff. Encadrement d'une double baguette végétalisée avec entrecolonne et rinceaux de feuilles au début de la première partie; bordure de double baguette prolongée de rinceaux de feuilles au début des deuxième et troisième parties ; trois grandes initiales ornées.

Copiste $R$. Première étape du texte, où quelques petits blancs ont été comblés ultérieurement ${ }^{16}$. Nombreuses préparations de rubriques, toutes, sauf une, écrites par le copiste ${ }^{17}$. Espaces de dimensions variables, souvent trop étroits, plus rarement trop larges, laissés pour l'inscription des rubriques; celles-ci empiètent donc fréquemment sur les marges ou l'entrecolonne. L'exécution des lettrines peintes a précédé celles des rubriques, qui les chevauchent parfois. Grande confusion dans la numérotation des chapitres. La préparation des tables des matières a constitué une opération distincte; chaque table de livre occupe un bifeuillet, deux de ceux-ci sont signés à la pointe sèche, alors que tous les autres cahiers sont signés à l'encre.

2. Modena, Biblioteca Estense $\boldsymbol{\alpha . N . 8 . 7 ^ { 1 8 }}$ (E). Parchemin, 295 x $217 \mathrm{~mm}$; justification 196,5 x $134 \mathrm{~mm}$; 32 ou 33 interlignes. (I) + $108+$ (I) ff. Encadrement

13 Voir G. Ouy et C. Reno, «Identification des autographes de Christine de Pizan», Scriptorium 34 (1980, fasc. 2), p. 221-38 et 3 pl., et J. C. Laidlaw, «Christine de Pizan - a Publishers's Progress », Modern Language Review 82 (1987), p. 37-75.

${ }^{14}$ Le manuscrit apparaît pour la première fois dans l'inventaire 1467-1469 des Ducs de Bourgogne; voir J. Barrois, Bibliothéque [sic] protypographique, ou librairies des fils du roi Jean, Charles V, Jean de Berri, Philippe de Bourgogne et les siens, Paris, Crapelet, 1830, p. $155, \mathrm{n}^{\circ}$ 984. Il appartint après à Charles-Quint (inventaire $1536 ; L F B M 1$, lxxxviii). En 1748, le ms. fut enlevé à la Bibliothèque de Bourgogne par Courchetet d'Esnans, commissaire de Louis XV et censeur royal; il est resté à Paris depuis. Voir A.-M. Legaré, «Les cent quatorze manuscrits de Bourgogne choisis par le comte d'Argenson pour le roi Louis XV. Édition de la liste de 1748 », Bulletin du bibliophile, 1998/2, p. 261, 298.

${ }^{15}$ Les parenthèses indiquent les feuillets de garde modernes.

${ }^{16}$ Voir infra «Corrections et compléments».

${ }^{17}$ La seule préparation de rubrique faite pas l'auteur, en cursive hâtive, se trouve au fol. $91^{\mathrm{v}}$.

${ }^{18}$ Le manuscrit apparaît pour la première fois dans la collection d'Ercole I, duc d'Este, (inventaire 1495, $\mathrm{n}^{\circ}$ 434). Voir G. Bertoni, La Biblioteca Estense e la coltura ferrarese ai tempi del Duca Ercole I (1471-1505), Torino, Ermanno Loescher, 1903, p. 28, 250. Il porte le numéro 153 dans la partie I de l'inventaire de 1756 de la collection des ducs de Modène ; voir B. Bacchini, Registro de Manuscritti [sic] della biblioteca del Ser. ${ }^{\text {mo }}$ Sig. ${ }^{r}$ Duca di Modena, fol. $6^{\mathrm{v}}$. Trois cotes du XVIII ${ }^{\mathrm{e}}$ s. paraissent sur la contregarde inférieure : «Ms. I.A.7», attribuée dans le catalogue de 1756-1759, «XI B 13 » (barrée) et «XX K 18175 », ces deux dernières attribuées par Carlo Ciocchi, bibliothécaire du palais ducal d'Este de 1794 à 1807. P. Loschi et G. Panelli, Catalogus Estensium manuscriptorum codicum veteri eorum 
d'une double baguette végétalisée avec entrecolonne et rinceaux de feuilles au début de la première partie ; bordure de double baguette prolongée de rinceaux de feuilles au début des deuxième et troisième parties ; trois grandes initiales ornées.

Copiste $R$. Étape plus tardive du texte, qui présente plusieurs modifications; comme dans B; quelques blancs - les mêmes - ont été laissés et comblés ultérieurement. Cet exemplaire présente des fautes d'inattention non corrigées; celles-ci - en particulier les mots omis - ne se retrouvent pas ailleurs ${ }^{19}$. Espaces de dimensions variables, souvent trop étroits, plus rarement trop larges, laissés pour les rubriques; empiètements plus fréquents que dans $\mathrm{B}$ sur les marges ou l'entrecolonne. Confusion dans la numérotation des chapitres, mais à un moindre degré que dans B. Nombreuses préparations de rubriques par le copiste. Les lettrines ont été peintes avant l'insertion des rubriques. La préparation des tables a constitué une opération distincte, chacune occupant un bifeuillet non signé.

3. Paris, BnF fr. 5025 ${ }^{20}$ (M). Parchemin, $286 \times 210 \mathrm{~mm}$; justification $196 \mathrm{x}$ $134 \mathrm{~mm}$; $33 \mathrm{ou}$, plus rarement, 32 interlignes entre les ff. 1-58; normalement 34 interlignes à partir du fol. 59; (III, papier) + II (parchemin) + 100 + I (parchemin) + (III, papier) ff. Encadrement d'une double baguette végétalisée avec entrecolonne et rinceaux de feuilles au début de la première partie; bordure de double baguette prolongée de rinceaux de feuilles au début des deuxième et troisième parties ; trois grandes initiales ornées.

Copiste $X$. Etape encore plus tardive du texte; les mêmes blancs que dans B et $\mathrm{E}$ ont été laissés et comblés ultérieurement. Certaines variantes du texte n'apparaissent que dans ce manuscrit ${ }^{21}$. Espaces de dimensions variables, souvent trop étroits, plus rarement trop larges, laissés pour les rubriques ; empiètements plus fréquents que dans B sur les marges ou l'entrecolonne. Aucune confusion dans la numérotation des chapitres. Aucune préparation de rubrique. Les lettrines ont été peintes avant l'insertion des rubriques. La préparation de la table de la première partie a constitué une opération distincte (le fol. 1 est indépendant). Il est probable que la table de la deuxième partie (ff. $25^{\mathrm{c}}-27^{\mathrm{a}}$ ) a été préparée de la même façon : on a

ordinatione servata; C. Ciocchi, Catalogo generale dei manoscritti del fondo estense diviso in cinque parti, sec. xviii.

${ }^{19}$ Signalons les erreurs suivantes : messire au lieu de mesure, couleur au lieu de couler et chartes au lieu de charetes (LFBM 1, 4, n. 1 et 95, n. $3 ;$ t. 2, 68, n. 3); $x x v i j^{e}$ au lieu de.$x x v j^{e}$ pour la date de la mort de Charles V (LFBM 2, 192, n. 3); un membre de phrase répété (t. 1, p. $232, \mathrm{n} .1$ ), et un $x$ manquant à ce qui devrait être $x x v j$, .xxvij. et $x x x i x .(t .2,74-75$ n. $5-6$; t. 2, 4, n. 4). Pour les lacunes, voir exemples : t. 1 : p. 7 , n. 5, p. 12, n. 2, p. 26, n. 3, p. 28, n. 1 , p. 38, n. 5 , p. 52 n. 2 , p. 98 , n. 3 , p. 219 , n. 5 , etc.

${ }^{20}$ Le manuscrit appartint au cardinal Mazarin (1602-1661) et entra à la bibliothèque du Roi en 1668. Voir BnF nouv. acq. fr. 5763, fol. $163^{\mathrm{r}}, \mathrm{n}^{\mathrm{o}} 397$. Au fol. $1^{\mathrm{d}}$, signature « Neufville» de Nicolas de Neufville, Seigneur de Villeroy (1542-1617).

${ }^{21}$ Voir $L F B M$ t. 1 : p. 34 , n. 3 , p. 36 , n. 2 , p. 66 , n. 4 , p. 71 , n. 3 , p. 73 , n. 6 , p. 75 , n. 4, p. 77 , n. 5 , p. 78 , n. 5 , p. 79 , n. 2 , p. 88 , n. 1 et 7 , p. 92 , n. 1 , p. 113 , n. 1 , p. 116 , n. 1 , p. 118 , n. 3 , p. 151 , n. 6 , p. 156 , n. 7 , p. 157 , n. 5 , p. 161 , n. 2 , etc. 
utilisé un bifeuillet sans signature (ff. 26-27). En revanche, la troisième table fait partie intégrante du neuvième cahier ${ }^{22}$.

4. Città del Vaticano, Biblioteca Apostolica Vaticana, Reg. lat. $920^{23}(\mathrm{C})^{24}$, Parchemin, 291 x $213 \mathrm{~mm}$; justification 196 x $133 \mathrm{~mm} ; 33 \mathrm{ou}$ - surtout à partir du fol. $60^{\mathrm{r}}-34$ interlignes. (II) $+112+$ (II) fol. Encadrement d'une double baguette végétalisée avec entrecolonne et rinceaux de feuilles au début de la première partie ; bordure de double baguette prolongée de rinceaux de feuilles au début des deuxième et troisième parties ; trois grandes initiales ornées.

Copiste $R$. Dernière étape du texte qui présente le plus grand nombre de changements ${ }^{25}$, mais les mêmes blancs que dans les trois autres mss. avaient été laissés, et comblés ultérieurement. Beaucoup de préparations de rubriques, toutes par $R$. Espaces de taille appropriée laissés pour les rubriques ; aucun blanc superflu ;

${ }^{22}$ Ce cahier (ff. $52-56,56^{\text {bis }}$, et 57-58) contient la conclusion de la partie II ainsi que la table des matières de la partie III.

${ }^{23}$ Le manuscrit appartint à Alexandre Petau, à qui Christine de Suède l'acheta en 1650. En 1689, à la mort de la reine, il passa à son héritier universel, le cardinal Decio Azzolini. Au décès de celui-ci, survenu moins de deux mois après, il fut vendu au pape Alexandre VIII (Pietro Ottoboni, 1689-1691). En 1797, le ms. figura parmi les «conquêtes artistiques» de l'armée républicaine française et fut rendu à la Vaticane en 1816. Voir Catalogus librorum manuscriptorum Ser ${ }^{\text {mae }}$ Christinae Reginae Sveciae qui Antverpae reperiuntur, Vat. lat. 8171, fol. 310 (olim fol. 138); Les Manuscrits de la reine de Suède au Vatican; Réédition du catalogue de Montfaucon et cotes actuelles, Città del Vaticano, Biblioteca Apostolica Vaticana, 1964 (Studi e Testi 238), p. 43 ; J. Bignami Odier, La Bibliothèque Vaticane de Sixte IV à Pie X, Città del Vaticano, Biblioteca Apostolica Vaticana, (Studi e Testi, 272), 1973, p. 185-89 ; M.-P. Laffitte, «La Bibliothèque nationale et les 'conquêtes artistiques' de la Révolution et de l'Empire; les manuscrits d'Italie (1796-1815) », Bulletin du bibliophile (1989/2), p. 285, 302-13.

${ }^{24} \mathrm{Il}$ existe deux copies sur papier de ce ms., exécutées toutes deux au XVII ${ }^{\mathrm{e}} \mathrm{s}$. La première, oeuvre de Théodore Godefroy, porte la cote BnF fr. 2862. La seconde, que Solente a examinée dans la collection de Thomas de Castelnau, appartint au chancelier de France H.-F. Daguesseau (1668-1751) et passa ensuite à son fils J.-B.-P. Daguesseau (†1784), au $4^{\mathrm{e}}$ comte d'Ashburnham, à Charles Fairfax Murray (acquisition 1901) et à Thomas de Castelnau (LFBM 1, xciv-xcv).

${ }^{25}$ Ces changements sont parfois des allègements : $L F B M 1:$ p. 88 , n. 10, p. 101, n. 1, p. 102, n. 1, p. 104, n. 1. La substitution de les plus sages à tous les sages (chap. I, 15 : LFBM 1, 39, n. 2) est indiscutablement une amélioration, de même que le remplacement de cellui par ledit captal $(1,88$, n. 4). Mais le plus souvent, les modifications apportées dans $\mathrm{C}$ alourdissent inutilement le texte: $L F B M 1:$ p. 23 , n. 2 , p. 39 , n. 1 , p. 43 , n. 5 , p. 44 , n. 5 , p. 57 , n. 3 , p. 62 , n. 2 , p. 64 , n. 4 , p. 73 , n. 2 et 5 , p. 74 , n. 6 , p. 78 , n. 3 , p. 87 , n. 4 , p. 88 , n. 3 , p. 88 , n. 5 et 6 , p. 92 , n. 4 , p. 112 , n. 3 etc. Deux erreurs voyantes introduites dans ce témoin sont signalées par Solente (LFBM 1, xcvii) : le remplacement de Poitiers par Cressy (II, 13, fol. $38^{\mathrm{d}}$ ) et de Bourgongne par Bretagne dans la rubrique du chap. II, 36 (fol. $59^{\mathrm{c}}$ ). Cette dernière pourrait, à la rigueur, être considérée comme une simple faute de copie, mais la première est plus difficile à expliquer. S'agit-il d'une étourderie du copiste, ou Christine aurait-elle introduit une erreur dans l'exemplar? 
rares empiètements sur les marges ou l'entrecolonne. Les rubriques ont été insérées avant l'exécution des lettrines. Les trois tables et le texte des trois parties sont copiés de façon continue : quatorze quaternions tous signés.

\section{Le texte}

Dans tous les témoins, le texte comporte trois parties divisées respectivement en 36,39 et 72 chapitres. Le texte varie davantage d'un manuscrit à l'autre que ne le signalent les variantes de l'édition critique, qui ne prend en compte que celles considérées par l'éditeur comme «les principales $»^{26}$. Aucun des quatre manuscrits n'a donc pu servir de modèle pour aucun des autres, et les copies ont été exécutées à partir d'un modèle qui était sans doute en même temps la maquette ${ }^{27}$. Ce modèle, auquel Christine travaillait sûrement encore quand la copie commença, pouvant à tout moment y apporter de nouveaux changements, devait se présenter sous forme de cahiers ou de liasses de papier à répartir entre les copistes (rien ne prouve que $P$ ait été tenu à l'écart). C'est seulement ainsi qu'il fut possible d'aboutir aussi rapidement: en effet, bien qu'encore d'autres copies, aujourd'hui disparues, notamment celle destinée à Jean de Berry, aient été aussi exécutées, il ne s'écoula qu'une quarantaine de jours entre l'achèvement de la composition et la présentation au duc de Berry de cet exemplaire perdu, qui n'était d'ailleurs probablement pas le premier manuscrit terminé.

Tout porte à croire que ce modèle était un véritable brouillon, ce qui expliquerait certaines erreurs commises par $R$, pourtant habitué à l'écriture hâtive de sa patronne ${ }^{28}$. L'exemplar devait également comporter au moins une lacune involontaire, car comment expliquer autrement, au premier chapitre, l'addition d'un mot indispensable dans la marge des manuscrits $\mathrm{B}$, E, et $\mathrm{M}^{29}$ ? Ceci montre, d'ailleurs, que toutes les fautes ne sont pas nécessairement imputables au copiste : il y en avait aussi, sans nul doute, dans le modèle, certaines ayant été corrigées par la suite, d'autres pas $^{30}$.

${ }^{26}$ LFBM 1, xcviii.

${ }^{27}$ La maquette devait être écrite sur papier, matériau bien moins cher que le parchemin ; voir G. Ouy, «Une Maquette de manuscrit à peintures [...]», Mélanges d'histoire du livre et des bibliothèques offerts à M. Frantz Calot, Paris, D'Argences, 1960, p. 43-51 + 1 pl.

${ }^{28}$ Alias au lieu de Bias (LFBM 2, 50, n. 1), et Breban pour Bourbon (2, 123, n. 5).

${ }^{29}$...hors le commun ordre de mes autres (choses) passees (I, $1:$ LFBM 1, 5). Dans le ms. E, le mot choses rajouté empiète sur l'encadrement et a donc été écrit après l'exécution de la décoration; il en va sans doute de même pour le ms. $\mathrm{M}$, où le rajout se trouve à l'extérieur de la bordure, et pour le ms. B. Le mot figure à sa place normale dans le ms. C ; il avait donc dû être ajouté au modèle avant la copie de ce manuscrit.

${ }^{30} \mathrm{Il}$ n'est pas toujours facile de distinguer les fautes commises par le copiste de celles que celui-ci a reprises de la maquette. Nous pouvons supposer le deuxième cas de figure pour les détails suivants: chevalerie et sagece manquent dans le chap. I, 13 (lacune dans les manuscrits $\mathrm{B}$ et $\mathrm{E}: L F B M 1,36, \mathrm{n} .3)$; puis manque dans I, 23 après oncques (LFBM 1, 61, n. 1); emploi fautif du singulier : peut (II, 2), doit (II, 4), voit (II, 39) : LFBM 1, p. 115, n. 3, p. 118, n. 3 et p. 242 , n. 3 . Une erreur concernant le mot precedent semble avoir persisté dans le modèle à travers les quatre exemplaires $(L F B M 1,12, \mathrm{n} .1)$. Si le ms. E porte cette leçon non corrigée, $\mathrm{M}$ présente une correction économique où seules les trois premières lettres sont grattées et 
Les blancs laissés dans les quatre manuscrits confirment que ce qui fut confié aux copistes était bien un texte inachevé, dans lequel l'auteur se proposait de compléter ou de préciser divers détails ${ }^{31}$.

En principe, outre les rectifications indispensables, tous les changements jugés souhaitables par l'auteur auraient dû être aussitôt reportés sur le modèle et se retrouver dans les copies ultérieures. C'est le cas la plupart du temps ${ }^{32}$, mais pas toujours, comme nous le verrons bientôt.

En dehors des modifications décidées par l'auteur, certaines variantes mineures que l'on relève d'un exemplaire à l'autre s'expliquent par les aléas de la copie. Ainsi, on peut attribuer à l'inattention la plupart des lacunes de nos quatre manuscrits - elles sont relativement plus nombreuses dans le ms. de Modène, comme le montrent les notes de l'édition critique. C'est, en revanche, le manque de place qui explique la chute des mots $C y$ dit au début de quelques rubriques, et même, dans le ms. de la Vaticane, le sacrifice d'un mot trop long, amoderement, qui figure bien dans la préparation de rubrique (III, 15) mais a disparu de la rubrique ${ }^{33}$.

Le nombre de leçons propres au ms. M mérite une attention particulière. Si on ne les trouve pas dans $\mathrm{C}$, qui lui est certainement postérieur, cela veut dire qu'elles n'étaient pas passées dans le modèle. Christine aurait-elle changé d'avis? Il est plus probable qu'elle ne pensait pas à reporter systématiquement sur l'exemplar tous les changements qu'elle apportait à son texte en cours de copie. A ce propos un cas significatif vaut d'être signalé : dans la relation de la campagne de Louis de Bourbon "en Barbarie» (II, 14), M se singularise: alors que, dans les deux précédents, l'événement est situé n'a mie long temps (B), puis n'a mie moult de temps (E), ici, l'auteur-copiste veut (fol. $38^{\mathrm{d}}$ ) ajouter une précision : $n$ 'a mie.....ans. Mais Christine oublie de faire la vérification, si bien que le nombre d'années est finalement laissé en blanc. Comme rien n'a changé sur le modèle, le manuscrit $\mathrm{C}$ revient tout naturellement à la leçon antérieure : n'a mie moult de temps.

remplacées par sus; C porte succedent écrit sur grattage, et B la variante ci aprés écrite sur grattage.

${ }^{31}$ Ces blancs (I, 6 et I, $8: L F B M 1,14$ et 1, 20-21) concernent la date de naissance de Charles (quantième et année) et son âge au moment où il monta sur le trône.

${ }^{32}$ Exemples de quelques changements incorporés au modèle avant l'exécution de la deuxième copie (E) : redoubté remplace redoubtable (I, $1: L F B M 1,5, \mathrm{n} .4)$; obviant ajouté devant a l'empire (LFBM 1, 13, n. 3); fors comme chose remplace fors choses (I, 6: LFBM 1, 16, n. 1); encore ajouté à la rubrique I, $10(L F B M 1,26)$; secours remplace sens (I, $14: L F B M 1$, 38 , n. 2) ; notables remplace nobles (I, $31: L F B M 1,87$, n. 3) ; grant ajouté devant aumosnier (I, $21: L F B M 1,90$, n. 6) ; affins remplace amis (II, 1: LFBM 1, 110, n. 3). Outre ces variantes introduites après la première copie, on trouve aussi des modifications apportées aux trois premiers manuscrits mais passées dans le texte du quatrième $(\mathrm{C})$, ce qui prouve qu'elles avaient été incorporées au modèle avant l'exécution de C. En plus du mot choses (voir note 29 supra), signalons la correction du mot i/ymaginable dans les trois premiers manuscrits devenue inutile dans le ms. C ( $L F B M 1,11,1.22)$, ou encore les mots fu coronné rajoutés dans les trois premiers exemplaires mais passés dans le texte de $C(1,20,1.13)$.

${ }^{33} L F B M 2,50$, n. 6. 


\section{Les rubriques}

Dans l'atelier de Christine comme partout ailleurs, les rubriques n'étaient exécutées qu'une fois achevée la copie du texte, afin d'éviter non seulement de constants changements de plume, mais sans doute aussi des taches d'encre rouge. Le copiste devait prendre soin de réserver la place nécessaire, et le texte de la future rubrique - en principe destiné à disparaître ultérieurement sous le grattoir - était inscrit dans la marge en petits caractères ${ }^{34}$.

Bien souvent (près de $40 \%$ des cas), dans trois de ces manuscrits, comme nous l'avons vu, l'espace prévu pour les rubriques avait été mal calculé3 ${ }^{35}$. D'ordinaire insuffisant, il est, plus rarement, trop vaste : c'est ainsi que la rubrique liminaire n'occupe guère plus de quatre des huit interlignes réservés; de même, certaines rubriques de la deuxième partie, celles qui concernent les ducs Philippe le Hardi (II, 13), et Louis d'Orléans (II, 16), comportent plus de blanc que d'écriture. Il en va tout autrement du ms. C, où l'espace a presque toujours été correctement évalués ${ }^{36}$.

Cette anomalie conduirait à se demander si le libellé des rubriques existait déjà au moment où les manuscrits $\mathrm{B}, \mathrm{E}$ et $\mathrm{M}$ furent mis en chantier. On pourrait en douter, en particulier, pour le ms. B car, si la plupart des rubriques y ont été copiées par $R$, cinq d'entre elles sont de $X^{37}$, et c'est la main $P$ - elle n'avait donc pas été tenue à l'écart - qui a écrit la rubrique liminaire au fol. $2^{\mathrm{a}}$ et ajouté les mots $E t$ premierement prologue .j. à la fin des rubriques qui introduisent les $2^{\mathrm{e}}$ et $3^{\mathrm{e}}$ parties (fol. $28^{\mathrm{a}}$ et $62^{\mathrm{a}}$ ). Au reste, le fait que - contrairement au processus normal - la décoration a précédé l'inscription des rubriques dans les trois premiers manuscrits paraîtrait confirmer l'hypothèse selon laquelle le texte des rubriques aurait été rédigé tardivement.

Mais cette hypothèse doit être écartée car, dans les trois premières copies, en dépit de la fréquente disproportion entre la place prévue et la longueur des rubriques, force est de constater que les espaces réservés pour leur inscription étaient presque identiques d'un manuscrit à l'autre. La mauvaise estimation des surfaces existait donc déjà dans la maquette.

De nombreuses préparations de rubriques, écrites de la même encre que le texte, subsistent dans les marges de $\mathrm{B}, \mathrm{E}$ et $\mathrm{C}$, les trois manuscrits copiés par $R^{38}$. Le

\footnotetext{
${ }^{34}$ Un détail dans le ms. E nous fait penser que, tout au moins dans ce ms., les rubriques étaient copiées après la constitution des cahiers et l'inscription des réclames. Ici, la réclame au fol. $9^{\mathrm{v}}$ : Cy dit de l'aage de meureté ne correspond pas au début du fol. $10^{\mathrm{r}}$, qui commence par la rubrique : Cy dit preuve par raison et exemples la noblece du corage du roy Charles xiiij, mais plutôt, à un mot près, à la rubrique du chap. I, 13: Cy dit encore de l'aage de meureté écrite au fol. $9^{\mathrm{r}}(L F B M 1,33)$.

${ }^{35}$ On relève un plus grand nombre d'espaces insuffisants dans $\mathrm{E}$ et $\mathrm{M}$ que dans B.

${ }^{36}$ Citons à titre d'exemple la rubrique liminaire, pour laquelle quatre interlignes ont été laissés et remplis, et les rubriques du II, 13 et 16 qui occupent exactement la place qui leur est laissée, respectivement un interligne et quart et un peu plus d'un interligne $(11 / 6)$.

${ }^{37}$ Il s'agit des rubriques des chapitres II, 10 (fol. $33^{\mathrm{d}}$ ) ; II, 25 (fol. $47^{\mathrm{d}}$ ) ; II, 35 (fol. $55^{\mathrm{d}}$ ) ; II, 36 (fol. $56^{\mathrm{b}}$ ) et III, 7 (fol. $66^{\mathrm{b}}$ ).

${ }^{38}$ Dans le ms. B, les préparations de rubriques subsistantes commencent au fol. $70^{\mathrm{v}}$ (III, 13) et vont jusqu'au pénultième chapitre (fol. $104^{\mathrm{v}}$ ). Dans $\mathrm{E}$, la première préparation de rubrique qui
} 
texte des rubriques figurait donc déjà, lui aussi, dans la maquette; mais tout porte à croire qu'il se présentait - ce qui serait d'ailleurs assez logique - sous la forme de préparations marginales, et que celles-ci étaient peut-être, comme plus tard dans les copies, écrites en tout petits caractères et chargées d'abréviations ${ }^{39}$. Cela ne facilitait pas l'évaluation correcte des espaces à réserver. Comme cela arrive souvent, c'est grâce à une erreur que l'on peut vérifier le bien-fondé de cette hypothèse : dans la marge inférieure du fol. $77^{\mathrm{r}}$ du manuscrit de la Vaticane, deux préparations de rubriques, celles des chapitres 18 et 19 sont interverties; il est évident que cela n'aurait pu se produire si, dans le modèle, chaque chapitre avait été précédé de sa rubrique.

Que, dans la dernière copie, $\mathrm{C}$, les espaces à réserver aient été correctement évalués prouve simplement que $R$ avait su tirer la leçon de ses deux premiers essais.

Nous savons maintenant que les rubriques existaient dès le début dans la maquette. Mais en allait-il de même pour les numéros qui devaient les accompagner dans les manuscrits? Il est permis d'en douter, étant donné la totale confusion qui règne au sein de la première copie, $\mathrm{B}$, tant dans la numérotation des préparations de rubriques que dans celle des rubriques elles-mêmes, et aussi bien en tête des chapitres que dans les tables des matières. Dans ce manuscrit, en effet, cinquantedeux préparations de rubriques subsistent entre les chapitres III, 13 et III, 71. Du chapitre 13 au chapitre 28 , chaque numéro de préparation de rubrique est corrigé par le rajout d'une unité, (celle du chapitre 29 étant rognée, on ne voit pas si elle comportait une correction). La préparation de rubrique du chapitre suivant (III, 30) porte le numéro .xxix. non corrigé. Le copiste continue à numéroter incorrectement jusqu'à 37 (qui correspond à III, 38), mais le numéro de la préparation de III, 39 est corrigé. À partir du chapitre 40, les numéros ont été ajoutés dans un second temps, d'abord à l'encre brun foncé (III, 40-45), puis à l'encre rouge (III, 47-69). Les numéros des chapitres 70-71, qui sont à l'encre brune, semblent avoir été faits dans un premier temps. La préparation de la rubrique du chapitre 46 a été ajoutée en cursive hâtive $\left(X^{\prime}\right)$ par Christine.

Dans cette première copie, ce n'est pas seulement pour les préparations, mais pour les rubriques elles-mêmes que se posent des problèmes dans la numérotation des chapitres de la troisième partie. Cette numérotation des rubriques ne suit d'ailleurs pas très fidèlement celle des préparations de rubriques : $R$ n'avait peut-être pas eu la patience d'essayer de débrouiller les choses et avait préféré tout recommencer. Quoi qu'il en soit, les rubriques des chapitres sont numérotées comme suit pour la partie III : 1-29, 29-55, 57, 58, 58, 59-66, 68-72. Quant à la table (fol. $61^{\text {a-d }}$ ), si elle donne finalement les vrais numéros, ce n'est que grâce à de nombreuses corrections apportées à partir du chapitre III, 39 .

La numérotation des chapitres a aussi posé quelques problèmes dans le ms. E. Dans la préparation de rubrique au fol. $77^{\mathrm{r}}, j$ est ajouté à .xxv. d'une encre différente, mais la rubrique correspondante porte le faux numéro $x v j$. et celle du chapitre suivant .xvij. (le numéro de la préparation de rubrique est rogné). Dans la préparation de rubrique du chapitre III, 58, à l'encre rouge, le numéro erroné .lix. a été

subsiste se trouve au fol. $77^{\mathrm{r}}$ (III, 26) et la dernière au fol. $103^{\mathrm{v}}$. Dans C, on voit des préparations de rubriques entre les folios $59^{\mathrm{v}}$ et $78^{\mathrm{v}}$.

${ }^{39}$ Merci à Carla Bozzolo qui nous a suggéré cette hypothèse. 
hâtivement corrigé en .lixviij. (le numéro a été également corrigé dans la rubrique). La préparation de rubrique du chapitre III, 63 a été corrigée par le rajout d'un $j$ à l'encre rouge. Le même numéro (.lxiij.) est répété dans la préparation de rubrique suivante, mais on trouve le numéro correct dans la rubrique correspondante. Au fol. $101^{\mathrm{v}}$, le numéro .lxvij. a été ajouté d'une encre plus foncée dans la préparation et corrigé dans la rubrique. Un $x$ a été oublié dans la préparation de rubrique du chapitre III, 69, mais la rubrique porte le numéro correct. De même, dans la préparation de rubrique au fol. $103^{\mathrm{v}}$, le numéro erroné .lviij. a été corrigé en .lix., mais le numéro de la rubrique était correct. À noter que les numéros des préparations de rubriques pour les chapitres III, 36-49 sont en chiffres arabes, contrairement à ce que l'on trouve partout ailleurs dans ce ms., comme dans les mss. $\mathrm{B}$ et $\mathrm{C}$.

Le ms. M, qui ne comporte pas de préparations de rubriques, ne présente aucune anomalie dans la numérotation des chapitres, ce qui semble bien être une nouvelle confirmation de la datation légèrement plus tardive que nous lui assignons par rapport aux mss. B et E. Quant au ms. C, le dernier des quatre, il ne présente, lui non plus aucune anomalie, ni dans les préparations de rubriques ni dans les rubriques.

L'examen attentif de ces quatre manuscrits permet donc de conclure que les numéros de chapitres n'avaient pas été prévus dès le début sur la maquette et qu'il a fallu procéder par essais et erreurs sur les deux premières copies avant de parvenir à une numérotation satisfaisante.

\section{Corrections et compléments}

Sans parler des corrections immédiates apportées en cours de copie, le plus souvent sur grattage, plus rarement par exponctuation, chaque manuscrit a fait l'objet d'une première campagne de correction. C'est la main $X$ qui y joue le rôle principal, travaillant à partir de préparations de correction; ces préparations, à en juger par celles que l'on a oublié de gratter, qui n'ont que partiellement disparu ou qui demeurent lisibles aux ultraviolets, étaient le plus souvent écrites dans la cursive hâtive $\left(X^{\prime}\right)$, mais parfois aussi - sans nul doute par inadvertance - dans la même écriture soignée que le texte ${ }^{40}$.

Outre ces corrections faites à chaque manuscrit individuellement, tous les quatre ont fait l'objet d'une dernière campagne destinée à leur apporter non seulement des corrections proprement dites, mais aussi des modifications ou des compléments identiques.

${ }^{40}$ Les corrections apportées par $X$ concernent des graphies, comme, dans B, fol. $6^{\text {d }}$ ligne 10 : [de]goust [é] et dans E, fol. $3^{\mathrm{c}}$ ligne 16 : [exerci]tees et [suppo]st, fol. $6^{\mathrm{a}}$ ligne 12 (I, $4:$ LFBM 1, 11, ligne 9 et début I, $9: L F B M 1,24]$. $X$ rajoute souvent des mots ou des passages oubliés, dont le plus long se trouve dans le ms. E, fol. $71^{\mathrm{r}}$, où la conclusion de III, 14 est écrite dans la marge inférieure depuis On raconte de Bias $(L F B M 2,50)$. Plus rarement, une intervention de $X$ constitue une variante, comme, dans $\mathrm{E}$, fol. $38^{\mathrm{r}}$, marge inférieure, Cartage à la place de Auffrique, leçon des autres manuscrits ( $L F B M 1,157$, n. 7). Il subsiste deux préparations de correction dans $\mathrm{M}:$ au fol. $74^{\mathrm{d}}$ : exept[é] (dernière lettre effacée lors de la correction); fol. $85^{\mathrm{d}}:$ et $p[$ rouffit $]$. 
L'aspect le plus remarquable de cette opération a consisté à combler des espaces réservés dans le texte, parfois avec, parfois sans préparation marginale. Les deux premiers, au chap. I, 6, concernent la date de naissance de Charles V: le jour saincte Agnès, xxj de janvier, en l'an de grace mille CCC XXXVI. Dans les quatre manuscrits, deux blancs avaient été laissés, le premier trop court, le deuxième un peu trop long, avant et après les mots en l'an de grace mille; ils ont été comblés avec une encre d'une teinte différente, de la main $X$ dans les mss. B, M et E, de la main $R$ dans le ms. C.

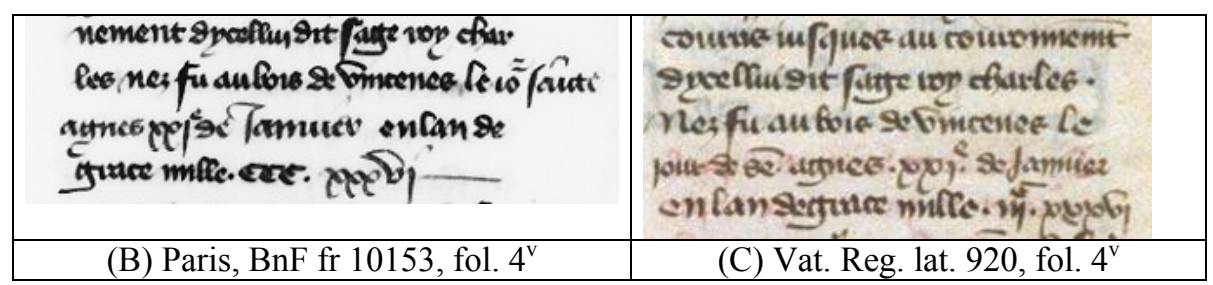

La troisième lacune concernait l'âge du roi lors de son couronnement. La précision.$x x v i j^{e}$. a été insérée dans un espace un peu trop large laissé dans les quatre manuscrits à la fin du passage le jour de la Trinité, en l'an de grace mille trois cens soixante et IIII, de sa nativité le ... ${ }^{41}$.

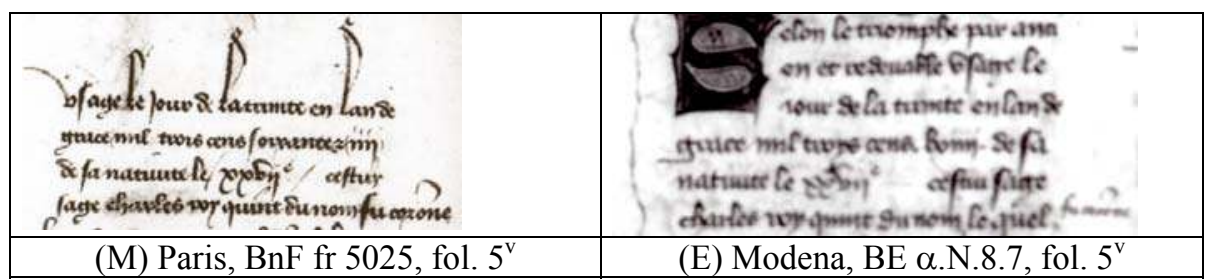

Une autre correction apportée aux quatre copies est le rajout du nom du comte de Tancarville devant celui de Jean de Hangest, sire d'Heuqueville au chapitre II, 17. Ce rajout pourrait étayer l'hypothèse de Suzanne Solente, qui pensait que le comte avait fourni à Christine certains détails sur les activités du roi défunt ${ }^{42}$. Dans le ms. M, le nom de Tancarville est suivi des mots seigneur veritable; dans $\mathrm{C}$, l'éloge s'amplifie : loyal conseillier preudomme et veritable... ${ }^{43}$. Au chapitre II, 13, Christine a décidé, après réflexion, de donner des précisions au sujet du mariage de deux filles de Philippe de Bourgogne, Catherine et Marie, le texte de l'ajout variant légèrement selon les copies : l'autre de ses filles au duc de Hausteriche et l'autre au

${ }^{41}$ LFBM 1, 20.

${ }^{42} L F B M 1$, lxxvii-lxxviii.

${ }^{43}$ Voir $L F B M 1,178$, n. 3. 
conte/jeune conte de Savoye $e^{44}$. Au chap. III, 25, le mot patrimoine a été remplacé par conquest sauf dans le ms. de Modène (M), copie qui semble avoir été faite dans la précipitation, où la leçon originale a dû être laissée par mégarde ${ }^{45}$. Au chap. II, 8 , une réflexion peu flatteuse au sujet des Anglais est rajoutée en marge, dans le ms. B sans préparation visible, avec préparation effacée dans les trois autres. Cette intervention est faite de la main $X$ dans les mss. B, E et M; dans le ms. C, elle est commencée par $R$ et terminée par $X$. Le texte de l'ajout est identique dans B et $\mathrm{C}$ : et mesmement que les Angloiz ne tenoyent pas bien les convenances comprises ou traictié, légèrement différent dans $\mathrm{E}$ (tenoyent mal les convenances) et plus encore dans M (mesmement que les Angloiz avoyent routes certaines couvenances [q]ue tenir devoyent).

\section{Décoration}

Comme on l'a signalé plus haut, la décoration a précédé la copie des rubriques dans B, E et M, mais l'a suivie dans C. Dans l'un et l'autre cas, c'est le même schéma qui a été utilisé par l'artiste ou les artistes ${ }^{46}$, schéma qui ne se retrouve dans la décoration d'aucun des autres manuscrits de Christine. Ce schéma consiste en un encadrement en diptyque à doubles baguettes au début de la première partie ${ }^{47}$ puis, au début des deuxième et troisième parties, en des doubles baguettes à rinceaux ${ }^{48}$. Une lettrine peinte d'entre quatre et demi et huit interlignes est mise au

${ }^{44}$ LFBM 1, 148, 1. 8-9.

${ }^{45}$ LFBM 2, 72, n. 3. Dans le ms. C, le mot patrimoine a été gratté dans la réclame du fol. $80^{\vee}$ mais est visible aux UV; le texte du fol. $81^{\text {a }}$ porte le mot correspondant conquest sur grattage. ${ }^{46}$ Le même ornemaniste aurait pu exécuter la décoration des mss. B, E et M, quoique celle de $\mathrm{M}$ paraisse moins soignée. Comme la décoration de $\mathrm{C}$ présente de légères variations, nous hésitons à nous prononcer sur ce point.

${ }^{47}$ L'encadrement est composé d'une double baguette inférieure et de trois doubles baguettes verticales à l'extrémité supérieure fourchue, prolongées en bas par des motifs végétaux stylisés. Les deux baguettes verticales principales sont formées d'une baguette plus épaisse à l'extérieur (3-4 mm) et d'une plus mince $(1 \mathrm{~mm})$ à l'intérieur, chacune étant divisée en trois sections en grenat et bleu avec rehauts de blanc qui alternent et se font contraste d'une baguette à l'autre; la baguette épaisse est entrecoupée de petits rectangles et triangles d'or. Les baguettes inférieures, plus minces ( $4 \mathrm{~mm}$. ensemble), n'ont que deux sections chacune. La baguette de l'entrecolonne est double elle aussi, mais encore plus étroite ( $3 \mathrm{~mm}$.) ; la tige de gauche est divisée en quatre sections (alternativement bleues et grenat), celle de droite est en or. Des rinceaux de feuilles poussant des extrémités des baguettes se prolongent dans les marges extérieure et inférieure. Dans le ms. C, les baguettes sont plus épaisses, mesurant 5 mm partout, sauf celle de l'entrecolonne.

${ }^{48}$ Baguette gauche : or bordé de noir ; baguette droite divisée en 3-4 sections alternativement bleues et grenat, rehaussées d'un filet blanc continu. Chaque extrémité de la baguette est fourchue, les extrémités finissant en un petit ovale brique bordé de blanc. De chaque bout de la baguette part un rinceau de feuilles qui se prolonge dans l'entrecolonne ou s'arrête avant. Les rinceaux, grenat ou bleus, sont ornés de feuilles de lierre lisses et dentées or, bleues et grenat, les deux dernières rehaussées de blanc. À gauche, brins de feuilles de lierre lisses or sur tige noire filiforme et brins colorés à deux feuilles chacun, lisses ou dentées. La décoration comprend des petits ronds d'or de diverses sortes. 
début de chaque partie de l'ouvrage. L'uniformité de la décoration se traduit même dans les motifs secondaires, tout à fait identiques dans les trois premiers manuscrits $(\mathrm{B}, \mathrm{E}, \mathrm{M})$, très légèrement différents dans le quatrième $(\mathrm{C})^{49}$.

\section{Conclusions}

L'examen de ces quatre manuscrits éclaire l'organisation du travail dans le petit atelier de Christine et nous permet de proposer quelques conclusions :

(1) Aucun des manuscrits de présentation n'a servi de modèle pour les autres; les copies ont toutes été faites à partir d'une maquette en constante évolution. On constate, certes, une dynamique visant à l'amélioration du texte; toutefois, l'introduction dans le ms. M de variantes qui ne se retrouvent pas ensuite dans $\mathrm{C}$ suggère, soit qu'à la réflexion, l'auteur ait préféré revenir au texte antérieur, soit - ce qui paraît bien plus probable - que les modifications n'étaient pas systématiquement reportées dans le modèle.

(2) De fait, même l'ordre normal de succession des différentes étapes de la production du manuscrit n'était pas systématiquement respecté. Si, dans le ms. C, la décoration a été exécutée après l'achèvement de la copie, il en va autrement dans les mss $\mathrm{B}, \mathrm{E}$ et $\mathrm{M}$, où la décoration a précédé non seulement l'insertion des rubriques, mais aussi, dans $\mathrm{E}$ et peut-être dans les deux autres, la copie des réclames et la composition du ms. en cahiers. Même, il n'est pas impossible que les rubriques aient été ajoutées alors que le volume était déjà relié.

(3) Les trois copistes que l'on voit à l'œuvre travaillent en collaboration: c'est, en l'occurrence, $R$ qui fait le gros du travail. $X$ copie un des manuscrits, écrit cinq rubriques dans un autre, complète un rajout, mais surtout apporte partout des corrections. Très actif ailleurs, $P$ n'intervient ici que de façon ponctuelle pour compléter quelques rubriques. La nature et la répartition de leurs interventions

\footnotetext{
${ }^{49}$ Les motifs secondaires comprennent : (1) brins à tige noire filiforme à une ou deux feuilles d'or, ceux à deux feuilles étant très asymétriques ; (2) rinceaux longs à tige noire filiforme aux feuilles d'or dentées et lisses avec des touches de bleu; dans B, E, et M, ce motif se trouve uniquement en marge gouttière, au milieu; (3) rinceaux courts et brins à tige noire et aux petites feuilles ovales bleues ou grenat rehaussées de blanc; (4) brins à tige bleue ou grenat rehaussée de blanc et ayant deux feuilles de couleur différente, le plus souvent bleu et grenat rehaussés de blanc; (5) petits ronds d'or variés. La plupart de ces motifs sont banals pour l'époque, sauf (2) et (3), qui, selon Inès Villela-Petit, le sont beaucoup moins. Dans le ms. C, un seul rinceau à tige colorée dans la marge gouttière, en haut. Les rinceaux en marge gouttière à tige noire filiforme et aux feuilles d'or rehaussés de bleu sont plus longs, et les feuilles d'or aux touches de bleu sont toutes lisses et agrémentées de petits cercles blancs dans la partie bleue. Ces rinceaux portent aussi des fleurs stylisées, formées d'un losange bleu aux côtés concaves ayant une croix blanche au milieu, le centre de la croix ornée d'un petit cercle d'or. Un petit rond d'or orne chaque côté du losange; (ces mêmes fleurs stylisées se retrouvent dans BnF fr. 1176). Nous remercions vivement Inès Villela-Petit de nous avoir aidés avec la terminologie de la décoration.
} 
respectives permettent de supposer que les trois personnes travaillaient ensemble dans un même local sous la direction de $X$.

(4) La décoration quasi-identique des quatre manuscrits montre que Christine avait sans doute confié cette tâche à un seul artisan ou atelier.

(5) Ces manuscrits témoignent de la rapidité avec laquelle Christine et ses auxiliaires parvenaient à diffuser ses ouvrages, mais cette rapidité n'allait pas nécessairement de pair avec la correction du texte.

Gilbert Ouy / Christine Reno CNRS / Vassar College/USA

avec la collaboration de James Laidlaw Université d’Édimbourg/ Écosse, RU 